\title{
SMALL VISCOSITY SHARP ESTIMATES FOR THE GLOBAL ATTRACTOR OF THE 2-D DAMPED-DRIVEN NAVIER-STOKES EQUATIONS *
}

\author{
A.A. ILYIN ${ }^{\dagger}$, A. MIRANVILLE $\ddagger$, AND E.S.TITI $\S$
}

\begin{abstract}
We consider in this article the damped and driven two-dimensional Navier-Stokes equations at the limit of small viscosity coefficient $\nu \rightarrow 0^{+}$. In particular, we obtain upper bounds of the order $\nu^{-1}$ on the fractal and Hausdorff dimensions of the global attractor for the system on the torus $T^{2}$, on the sphere $S^{2}$ and in a bounded domain. Furthermore, in the case of the torus, we establish a lower bound of the order $\nu^{-1}$. This sharp estimate is remarkably smaller than the well established sharp bound for the dimension of the global attractor of the Navier-Stokes equations on the torus $T^{2}$, which is of the order $\nu^{-4 / 3}$. This means that the damping/friction term plays a significant role in reducing the number of degrees of freedom in this two-dimensional model. This, we believe, is done by dissipating the energy at the large spatial scales which is transferred to these scales via the inverse cascade mechanism. Finally, we remark that the system of equations studied here is related to the Stommel-Charney barotropic ocean circulation model of the gulf stream.
\end{abstract}

Key words. Kolmogorov flows, attractor dimension, Navier-Stokes equations, StommelCharney model, gulf stream, barotropic ocean circulation model.

AMS subject classifications: 35Q30, 76D05, 76U05, 76E20, 86A05, 86A10.

\section{Introduction}

The two-dimensional Navier-Stokes system

$$
\begin{aligned}
\partial_{t} u+\sum_{i=1}^{2} u^{i} \partial_{i} u & =\nu \Delta u-\nabla p+f \\
\operatorname{div} u & =0 \\
u(0) & =u_{0}
\end{aligned}
$$

rightfully occupies one of the central places in the theory of global attractors for dissipative partial differential equations [2], [11], [26], [31], [33], [35]. Here, $u$ is the velocity vector field, $p$ is the pressure, $\nu>0$ is the viscosity coefficient, and $f$ is the forcing term.

System (1.1), supplemented with appropriate boundary conditions (Dirichlet or space-periodic), possesses the global attractor $\mathcal{A}$ in the corresponding phase space. Concerning the Navier-Stokes system (1.1) in a bounded domain $\Omega$ with the Dirichlet boundary conditions $\left.u\right|_{\partial \Omega}=0$, we have the following estimate for the Hausdorff and fractal dimensions of the attractor [35]:

$$
\operatorname{dim}_{H} \mathcal{A} \leq \operatorname{dim}_{F} \mathcal{A} \leq c(\Omega) G, \quad G=\frac{\|f\|}{\nu^{2} \lambda_{1}},
$$

\footnotetext{
*Received: May 4, 2004; accepted: July 9, 2004. Communicated by Leslie Smith.

$\dagger$ Keldysh Institute of Applied Mathematics, Russian Academy of Sciences, Miusskaya Sq. 4, 125047 Moscow, Russia (ilyin@spp.keldysh.ru).

$\ddagger$ Université de Poitiers, Laboratoire de Mathématiques et Applications, UMR CNRS 6086, SP2MI, Téléport 2, 86962 Chasseneuil Futuroscope Cedex, France (miranv@math.univ-poitiers.fr).

$\S$ Department of Mathematics and Department of Mechanical and Aerospace Engineering, University of California, Irvine, California 92697, USA (etiti@math.uci.edu). Also: Department of Computer Science and Applied Mathematics, Weizmann Institute of Science, P.O. Box 26, Rehovot, 76100, Israel (edriss.titi@weizmann.ac.il).
} 
where $\lambda_{1}$ is the first eigenvalue of the Stokes operator, $\|f\|=\|f\|_{L_{2}(\Omega)}$, and $c(\Omega)$ is a dimensionless constant. The best to date explicit form of this estimate was found in $[8]$ :

$$
\operatorname{dim}_{F} \mathcal{A} \leq \frac{1}{\sqrt{2} \pi}\left(\lambda_{1}|\Omega|\right)^{1 / 2} \frac{\|f\|}{\lambda_{1} \nu^{2}} \leq \frac{1}{2 \pi^{3 / 2}} \frac{\|f\||\Omega|}{\nu^{2}},
$$

where $|\Omega|$ is the area of $\Omega$.

In the space-periodic case $\left(x \in[0,2 \pi L]^{2}\right)$, the estimate for the dimension of the global attractor can be significantly improved [11], [12], [35]:

$$
\operatorname{dim}_{F} \mathcal{A} \leq c G^{2 / 3}(\ln (1+G))^{1 / 3} .
$$

Moreover, it was shown in [28] that the following lower bound holds for the Kolmogorov forcing:

$$
\operatorname{dim}_{F} \mathcal{A} \geq c^{\prime} G^{2 / 3}
$$

which shows that estimate (1.2) is sharp up to a logarithmic correction. Estimate (1.2) also holds for the Navier-Stokes system on a two-dimensional compact manifold (for example, $S^{2}$ ) and in a bounded domain with the so-called free boundary conditions [22]. We also note that, so far, no lower bounds are known for system (1.1) with Dirichlet boundary conditions.

In this work, we shall deal with the following system:

$$
\begin{aligned}
\partial_{t} u+\sum_{i=1}^{2} u^{i} \partial_{i} u+\mathbf{k} l \times u & =-\mu u+\nu \Delta u-\nabla p+f, \\
\operatorname{div} u & =0, \\
u(0) & =u_{0},
\end{aligned}
$$

where, preserving the previous notation, we have the additional drag/friction term $-\mu u$ on the right-hand side and the Coriolis acceleration on the left-hand side. Here, $\mathbf{k}$ is the vertical unit vector and $l=l_{0}+\beta x_{2}$ is the Coriolis parameter in the $\beta$-plane approximation. System (1.3), with $\mu>0$ and $\nu \geq 0$, has important applications in geophysical hydrodynamics (see, for example, [13], [30].) The drag/friction term $-\mu u$, where $\mu$ is the Rayleigh friction coefficient (or the Ekman pumping/dissipation constant), models the bottom friction in two-dimensional oceanic models (when the system is considered in a bounded domain; in that case, the system is called the viscous Charney-Stommel barotropic ocean circulation model of the gulf stream) or the Rayleigh friction in the planetary boundary layer (for two-dimensional atmospheric models on the sphere or with space-periodic boundary conditions). Existence and uniqueness results for the stationary problem and also results on the stability of stationary solutions for (1.3) with $\nu=0$ can be found in [3], [32], [36]. Weak global attractors for this system, with $\nu=0$, were constructed in [21], [18] and [19] (see also [5]). An investigation of the bifurcation diagram and other related dynamical properties of the system (1.3) are reported in [6] (see also references therein).

Whether the weak global attractor of the system (1.3), when $\nu=0$, is finite or infinite dimensional is an open question. In this work, however, we shall deal with the case when $\mu>0$ is arbitrary but fixed and $\nu>0$ is small, $\nu \rightarrow 0^{+}$. One can think of this system as the Navier-Stokes perturbation (or viscous perturbation) of the dampeddriven two-dimensional Euler equations with rotation. Unfortunately, this work does 
not give us any clue about the more challenging case when $\nu=0$ and $\mu>0$. This is because attractors are usually not robust under small perturbations in parameters. It is worth mention that similar to the two-dimensional Navier-Stokes equations one can show the existence of finite number of degrees of freedom (determining modes, nodes, etc..) for the system (1.3) when $\nu>0$ (see, for example, [4], [9], [14], [15], [16], [24], [25], [31] and references therein). Here again it is not known whether the system (1.3), with $\nu=0$, has finite number of degrees of freedom (determining modes, nodes, etc...).

In section 1, we establish explicit upper bounds on the fractal dimension of the global attractor for the space-periodic system (1.3) and also explicit lower bounds when $f$ is the Kolmogorov forcing. We observe that both the upper and lower bounds are of order $\nu^{-1}$ as $\nu \rightarrow 0^{+}$, see (2.43). We recall that, for the classical Navier-Stokes equations, we have an upper bound of the order $\nu^{-2}$ for Dirichlet boundary conditions and of order $\nu^{-4 / 3}(\ln (1 / \nu))^{1 / 3}$ for space-periodic boundary conditions. Thus, for any $\mu>0$, and even in the space-periodic case, we have a much smaller estimate for the global attractor, which is of the order $\nu^{-1}$ as $\nu \rightarrow 0^{+}$. Moreover, this estimate is sharp. This observation has significant physical implications concerning two-dimensional turbulence. The damping/drag/friction plays an important role in reducing the number of degrees of freedom in this two-dimensional model. This, we believe, is done by dissipating the energy at the large spatial scales that is transferred to these large scales via the inverse cascade mechanism, a characteristic of two-dimensional turbulence. This interesting physical observation will be the subject of future work.

In section 2, we obtain explicit upper bounds on the fractal dimension of the global attractor for system (1.3) on the rotating sphere and for the system in a bounded domain with free boundary conditions.

Finally, in the Appendix in section 3, we recall the Lieb-Thirring inequalities for space-periodic vector functions.

\section{Two-sided estimates for the space-periodic model}

We consider in this section system (1.3) with space-periodic boundary conditions. We assume in what follows that the Rayleigh friction coefficient $\mu>0$ is arbitrary but fixed, while the viscosity coefficient $\nu>0$ is small so that

$$
\frac{\mu}{\nu L^{2}} \geq 1
$$

However, condition (2.1) is used only for obtaining the lower bounds. The upper estimates derived in this section are valid for any combination of the parameters. Finally, the spatial variable $x$ belongs to the two-dimensional torus $T^{2}=[0,2 \pi L]^{2}$.

Using the standard notation (see, for instance, [11], [35]), we denote by $P$ the orthogonal projection in $\left(L_{2}\left(T^{2}\right)\right)^{2} \cap\left\{u: \int_{T^{2}} u d x=0\right\}$ onto the Hilbert space $H$ which is the closure in $\left(L_{2}\left(T^{2}\right)\right)^{2}$ of the set of smooth solenoidal periodic vector functions with mean value zero. Applying $P$ to the first equation in (1.3), we obtain

$$
\partial_{t} u+B(u, u)+K u+\nu A u=-\mu u+f, \quad u(0)=u_{0},
$$

where $A=-P \Delta$ is the Stokes operator, $B(u, v)=P\left(\sum_{i=1}^{2} u^{i} \partial_{i} v\right)$ is the nonlinear term, $K u=P(\mathbf{k} l \times u)$, and $f \in H$.

It is standard to prove (see, for instance, [2], [11], [26], [35]) that, for every initial value $u_{0} \in H$, equation (2.2) has a unique solution $u(t) \in H$ generating thereby the 
semigroup $S_{t}: H \rightarrow H, S_{t} u_{0}=u(t)$. The semigroup $S_{t}$ has a global attractor $\mathcal{A} \Subset H$. Furthermore, if $f \in H$, then $\mathcal{A}$ is bounded in the Sobolev space $H_{2}$. We assume in what follows that $f \in H_{1}$, so that $\|\operatorname{rot} f\|$ is finite.

We first obtain some a priori estimates for the solutions $u(t)$. We shall use the following important identity (see, for instance, [11], [35]):

$$
(B(u, u), A u)=0, \quad u \in H_{2} \cap H .
$$

For the sake of completeness, we give an invariant proof of (2.3) (in the sense that it also works for the sphere and a bounded domain with free boundary conditions) [22]. Since $\sum_{i=1}^{2} u^{i} \partial_{i} u=\nabla u^{2} / 2-u \times \operatorname{rot} u$ and, in the periodic case, $A u=\operatorname{rot}(\operatorname{rot} u)$, we introduce the stream function $\psi$, so that $u=\left\{-\partial_{2} \psi, \partial_{1} \psi\right\}$, and obtain

$$
(B(u, u), A u)=(\operatorname{rot} u \times u, \operatorname{rot}(\operatorname{rot} u))=(\operatorname{rot}(\operatorname{rot} u \times u), \operatorname{rot} u)=(J(\psi, \Delta \psi), \Delta \psi)=0,
$$

where the Jacobian operator $J$ is defined below in (2.16). In addition to the trivial identity $(K u, u)=0$, we also have (taking into account that $\left.l=l_{0}+\beta x_{2}\right)$

$$
(K u, A u)=(P(\mathbf{k} l \times u), \operatorname{rot}(\operatorname{rot} u))=(\operatorname{rot}(\mathbf{k} l \times u), \operatorname{rot} u)=\beta\left(\partial_{1} \psi, \Delta \psi\right)=0 .
$$

Multiplying (2.2) by $A$ u, using the above identity and (2.3) and integrating by parts, we obtain

$$
\begin{array}{r}
\partial_{t}\|\operatorname{rot} u\|^{2}+2 \nu\|A u\|^{2}+2 \mu\|\operatorname{rot} u\|^{2} \\
=2(f, A u)=2(\operatorname{rot} f, \operatorname{rot} u) \leq \mu\|\operatorname{rot} u\|^{2}+\mu^{-1}\|\operatorname{rot} f\|^{2} .
\end{array}
$$

Dropping the second term in the left-hand side, we have

$$
\partial_{t}\|\operatorname{rot} u\|^{2}+\mu\|\operatorname{rot} u\|^{2} \leq \mu^{-1}\|\operatorname{rot} f\|^{2}
$$

and, by Gronwall's inequality, we find that

$$
\|\operatorname{rot} u(t)\|^{2} \leq\|\operatorname{rot} u(0)\|^{2} e^{-\mu t}+\mu^{-2}\|\operatorname{rot} f\|^{2}\left(1-e^{-\mu t}\right),
$$

so that we have, on the attractor $\left(u_{0} \in \mathcal{A}\right)$,

$$
\|\operatorname{rot} u(t)\| \leq \frac{\|\operatorname{rot} f\|}{\mu} .
$$

2.1. Upper bounds on the dimension of the global attractor. We now estimate the dimension of the global attractor $\mathcal{A}$. To take advantage of the fact that estimate (2.4) is independent of $\nu$, we estimate the dimension of $\mathcal{A}$ in the phase space $H$.

For a solution $u(t)$ lying on the attractor $\left(u_{0} \in \mathcal{A}\right)$, we consider the linearized equation

$$
\partial_{t} U=-\nu A U-K U-\mu U-B(U, u(t))-B(u(t), U)=: \mathcal{L}\left(t, u_{0}\right) U, \quad U(0)=\xi,
$$

and estimate the $m$-trace of the operator $\mathcal{L}$. Let $U_{i}(t)$ be the solution of $(2.5)$ with $U_{i}(0)=\xi_{i}, i=1, \ldots, m$. We denote by $Q_{m}(t)$ the orthogonal projection in $H$ onto 
$\operatorname{Span}\left\{U_{1}(t), \ldots, U_{m}(t)\right\}$. Since $U_{i}(t) \in H_{1}$ for $t>0$, we denote by $v_{i}=v_{i}(t) \in H \cap H_{1}$, $i=1, \ldots, m$, an orthonormal (in $H$ ) basis of $\operatorname{Span}\left\{U_{1}(t), \ldots, U_{m}(t)\right\}$. Integrating by parts, using the identities $\left(B\left(u(t), v_{j}\right), v_{j}\right)=0$ and $\left(K v_{j}, v_{j}\right)=0$, the orthonormality of the $v_{j}$ s and the inequality (see [8] or Lemma 3.2 below)

$$
\sum_{i, k=1}^{2} v^{k}(x) \partial_{k} u^{i}(x) v^{i}(x) \leq 2^{-1 / 2}|v(x)|^{2}|\nabla u(x)|,
$$

we have

$$
\begin{aligned}
& \operatorname{Tr}\left[\mathcal{L}\left(t, u_{0}\right) \circ Q_{m}(t)\right]=\sum_{j=1}^{m}\left(\mathcal{L}\left(t, u_{0}\right) v_{j}, v_{j}\right)= \\
& -\nu \sum_{j=1}^{m}\left\|\operatorname{rot} v_{j}\right\|^{2}-\mu m+\int_{T^{2}} \sum_{j=1}^{m} \sum_{i, k=1}^{2} v_{j}^{k}(x) \partial_{k} u^{i}(x) v_{j}^{i}(x) d x \leq \\
& -\nu \sum_{j=1}^{m}\left\|\operatorname{rot} v_{j}\right\|^{2}-\mu m+2^{-1 / 2} \int_{T^{2}} \rho(x)|\nabla u(x)| d x \leq \\
& -\nu \sum_{j=1}^{m}\left\|\operatorname{rot} v_{j}\right\|^{2}-\mu m+2^{-1 / 2}\|\rho\|\|\operatorname{rot} u(t)\|,
\end{aligned}
$$

where

$$
\rho(x)=\sum_{j=1}^{m}\left|v_{j}(x)\right|^{2}, \quad(\nabla u(x))^{2}=\sum_{i, k=1}^{2}\left(\partial_{k} u^{i}(x)\right)^{2}, \quad\|\nabla u\|=\|\operatorname{rot} u\| .
$$

Next, we note that

$$
\sum_{j=1}^{m}\left\|\nabla v_{j}\right\|^{2}=\sum_{j=1}^{m}\left\|\operatorname{rot} v_{j}\right\|^{2} \geq \lambda_{1}+\cdots+\lambda_{m},
$$

where $L^{-2}=\lambda_{1} \leq \lambda_{2} \leq \ldots$ are the eigenvalues of the Stokes operator $A$ which, in the space-periodic case, coincide with the eigenvalues of the scalar Laplacian $-\Delta$ in the space $L_{2}\left(T^{2}\right) \cap\left\{\varphi: \int_{T^{2}} \varphi(x) d x=0\right\}$. We use the fact that, in two space dimensions, $\lambda_{j} \geq c_{1} \lambda_{1} j$ and, hence,

$$
\sum_{j=1}^{m} \lambda_{j} \geq \lambda_{1} c_{\mathrm{T}^{2}-\mathrm{spec}} m^{2}
$$

where we can take (see Proposition 1 in section 3)

$$
c_{1}=\frac{1}{4}, \quad c_{\mathrm{T}^{2}-\mathrm{spec}}=\frac{1}{8} .
$$

We also recall the two-dimensional Lieb-Thirring inequality:

$$
\|\rho\|^{2}=\int_{T^{2}}\left(\sum_{j=1}^{m}\left|v_{j}(x)\right|^{2}\right)^{2} d x \leq c_{\mathrm{T}^{2}-\mathrm{LT}} \sum_{j=1}^{m}\left\|\operatorname{rot} v_{j}\right\|^{2},
$$


where we can take the following explicit upper estimate for $c_{\mathrm{T}^{2}-\mathrm{LT}}$ (see Theorem 4.3):

$$
c_{\mathrm{T}^{2}-\mathrm{LT}} \leq \frac{6}{\pi}
$$

The main tool in the estimates of the attractor's dimension are the numbers $q(m)$ (the sums of the first global Lyapunov exponents) and the trace formula, see [10], [11], [35]. By the trace formula and (2.7), (2.9), (2.4), we have

$$
\begin{aligned}
& q(m) \leq \limsup _{t \rightarrow \infty} \sup _{u_{0} \in \mathcal{A}} \sup _{\substack{\xi_{i} \in H \\
i=1, \ldots, m}} \frac{1}{t} \int_{0}^{t} \operatorname{Tr}\left[\mathcal{L}\left(\tau, u_{0}\right) \circ Q_{m}(\tau)\right] d \tau \leq \\
& -\nu \sum_{j=1}^{m}\left\|\operatorname{rot} v_{j}\right\|^{2}-\mu m+2^{-1 / 2}\left(c_{\mathrm{T}^{2}-\mathrm{LT}} \sum_{j=1}^{m}\left\|\operatorname{rot} v_{j}\right\|^{2}\right)^{1 / 2} \frac{\|\operatorname{rot} f\|}{\mu} \leq \\
& -\frac{\nu}{2} \sum_{j=1}^{m}\left\|\operatorname{rot} v_{j}\right\|^{2}-\mu m+\frac{c_{\mathrm{T}^{2}-\mathrm{LT}}}{4 \nu} \frac{\|\operatorname{rot} f\|^{2}}{\mu^{2}} \leq \\
& \leq g(m):=-\frac{\nu}{2} \lambda_{1} c_{\mathrm{T}^{2}-\mathrm{spec}} m^{2}-\mu m+\frac{c_{\mathrm{T}^{2}-\mathrm{LT}}}{4 \nu} \frac{\|\operatorname{rot} f\|^{2}}{\mu^{2}} .
\end{aligned}
$$

Let $d_{*}$ be the unique positive root of the quadratic equation $g(d)=0$. Then both the Hausdorff (see [35]) and the fractal (see [7], [8]) dimensions satisfy the estimate

$$
\operatorname{dim}_{H} \mathcal{A} \leq \operatorname{dim}_{F} \mathcal{A} \leq d_{*} .
$$

Dropping the second term in the right-hand side of the above inequality for $q(m)$, we find

$$
q(m) \leq g_{1}(m):=-\frac{\nu}{2} \lambda_{1} c_{\mathrm{T}^{2}-\mathrm{spec}} m^{2}+\frac{c_{\mathrm{T}^{2}-\mathrm{LT}}}{4 \nu} \frac{\|\operatorname{rot} f\|^{2}}{\mu^{2}}
$$

Hence,

$$
\operatorname{dim}_{H} \mathcal{A} \leq \operatorname{dim}_{F} \mathcal{A} \leq d_{*}<d_{*}^{1}=\left(\frac{c_{\mathrm{T}^{2}-\mathrm{LT}}}{2 c_{\mathrm{T}^{2}-\mathrm{spec}}}\right)^{1 / 2} \frac{\|\operatorname{rot} f\|}{\nu \mu \lambda_{1}^{1 / 2}}
$$

where $g_{1}\left(d_{*}^{1}\right)=0$. Dropping the first term, we find

$$
q(m) \leq g_{2}(m):=-\mu m+\frac{c_{\mathrm{T}^{2}-\mathrm{LT}}}{4 \nu} \frac{\|\operatorname{rot} f\|^{2}}{\mu^{2}}
$$

Accordingly,

$$
\operatorname{dim}_{H} \mathcal{A} \leq \operatorname{dim}_{F} \mathcal{A} \leq d_{*}<d_{*}^{2}=\frac{c_{\mathrm{T}^{2}-\mathrm{LT}}}{4} \frac{\|\operatorname{rot} f\|^{2}}{\nu \mu^{3}}
$$

where $g_{2}\left(d_{*}^{2}\right)=0$. 
Taking into account (2.8), (2.10) and the fact that $\lambda_{1}=L^{-2}$, we obtain the following theorem.

THEOREM 2.1. Equation (1.3) has a global attractor $\mathcal{A}$ whose Hausdorff and fractal dimensions satisfy the following upper bound:

$$
\operatorname{dim}_{H} \mathcal{A} \leq \operatorname{dim}_{F} \mathcal{A} \leq \min \left(2\left(\frac{6}{\pi}\right)^{1 / 2} \frac{\|\operatorname{rot} f\| L}{\nu \mu}, \frac{3}{2 \pi} \frac{\|\operatorname{rot} f\|^{2}}{\nu \mu^{3}}\right) .
$$

2.2. Lower bounds on the dimension of the global attractor. We now deduce a sharp lower bound on the dimension of the global attractor, our main assumption being that condition (2.1) is satisfied. In other words, we study the behavior of the dimension when $\mu>0$ is arbitrary and fixed and $\nu \rightarrow 0^{+}$.

We first go over to a scalar vorticity equation. Using the condition $\operatorname{div} u=0$, we introduce the stream function $\psi$, so that $u=\mathbf{k} \times \nabla \psi=-\operatorname{rot} \psi=\left\{-\partial_{2} \psi, \partial_{1} \psi\right\}$. The choice of $\psi$ is fixed by the condition $\int_{T^{2}} \psi d x=0$. We substitute this into the first equation in (1.3) and apply the rot operator. We then obtain a scalar equation:

$$
\partial_{t} \Delta \psi-\nu \Delta^{2} \psi+\mu \Delta \psi+J(\psi, \Delta \psi+l)=F=\operatorname{rot} f .
$$

We derive our lower bounds without the Coriolis force and set $l=0$. Next, setting $\varphi=\Delta \psi$, we obtain

$$
\partial_{t} \varphi-\nu \Delta \varphi+\mu \varphi+J\left(\Delta^{-1} \varphi, \varphi\right)=F,
$$

where

$$
J(a, b)=n \times \nabla a \cdot \nabla b=\partial_{1} a \partial_{2} b-\partial_{2} a \partial_{1} b .
$$

Since a global attractor is a maximal strictly invariant compact set, it follows that it contains the unstable manifold of every stationary point $\bar{\varphi}, M^{\mathrm{u}}(\bar{\varphi}) \subset \mathcal{A}$, that is, the invariant manifold along which the solutions of (2.15) tend exponentially to the stationary point $\bar{\varphi}$ as $t \rightarrow-\infty$. Since $\nu>0$, the local invariant manifold near the stationary point $\bar{u}$ can be constructed in the framework of the general theory of invariant manifolds for parabolic equations [2], [17].

In our analysis, we shall use the well-known family of Kolmogorov flows [2], [23], [28], [29], [37] (see also [38]).

We set $L=1$. (This involves no loss of generality and simplifies the notation of the Fourier series below.) As in [23], for a (large) parameter $s$, to be determined later, we consider the following family of right-hand sides $f$ :

$$
f=f_{s}=\left\{\begin{array}{l}
f_{1}=\frac{1}{\sqrt{2} \pi} \nu^{2} \lambda s^{2} \sin s x_{2}, \\
f_{2}=0
\end{array}\right.
$$

where $\lambda=\lambda(s)$ is a parameter to be chosen later. Then 


$$
\operatorname{rot} f_{s}=F_{s}=-\frac{1}{\sqrt{2} \pi} \nu^{2} \lambda s^{3} \cos s x_{2},
$$

so that

$$
\left\|\operatorname{rot} f_{s}\right\|=\nu^{2} \lambda s^{3}
$$

We now consider the stationary equation (2.15) with right-hand side (2.18):

$$
-\nu \Delta \varphi+\mu \varphi+J\left(\Delta^{-1} \varphi, \varphi\right)=-\frac{1}{\sqrt{2} \pi} \nu^{2} \lambda s^{3} \cos s x_{2}
$$

and look for a solution of this equation of the form

$$
\varphi=\varphi_{s}=-\frac{1}{\sqrt{2} \pi} \nu \lambda s K \cos s x_{2}, \quad K=K(s, \mu, \nu) .
$$

Since $\varphi_{s}$ only depends on $x_{2}$, it follows that $J\left(\Delta^{-1} \varphi_{s}, \varphi_{s}\right) \equiv 0$ and it is straightforward to see that, for

$$
K(s, \mu, \nu)=\frac{s^{2}}{s^{2}+\mu / \nu},
$$

the function $\varphi_{s}$ defined by (2.21) is a solution of equation (2.20).

We consider the eigenvalue problem for the equation linearized around the stationary solution $\varphi_{s}$ :

$$
\mathcal{L}_{\varphi_{s}} \varphi=J\left(\Delta^{-1} \varphi_{s}, \varphi\right)+J\left(\Delta^{-1} \varphi, \varphi_{s}\right)-\nu \Delta \varphi+\mu \varphi=-\sigma \varphi .
$$

The dimension of the unstable eigenspace with $\operatorname{Re} \sigma>0$ will bound from below the dimension of the global attractor $\mathcal{A}$.

Substituting the Fourier representation of $\varphi$,

$$
\begin{gathered}
\varphi=\frac{1}{\sqrt{2} \pi} \sum_{k \in \mathbb{Z}_{+}^{2}}\left(a_{k} \cos k x+b_{k} \sin k x\right), \\
\mathbb{Z}_{+}^{2}=\left\{k \in \mathbb{Z}_{0}^{2}, \quad k_{1} \geq 0, k_{2} \geq 0\right\} \cup\left\{k \in \mathbb{Z}_{0}^{2}, \quad k_{1} \geq 1, \quad k_{2} \leq 0\right\}, \quad \mathbb{Z}_{0}^{2}=\mathbb{Z}^{2} \backslash\{0\},
\end{gathered}
$$

into (2.23) and using the equality $J(a, b)=-J(b, a)$, we obtain

$$
\begin{aligned}
& \frac{\lambda K s}{\sqrt{2} \pi} \sum_{k \in \mathbb{Z}_{+}^{2}}\left(\frac{1}{s^{2}}-\frac{1}{k^{2}}\right) J\left(\cos s x_{2}, a_{k} \cos k x+b_{k} \sin k x\right)+ \\
& +\sum_{k \in \mathbb{Z}_{+}^{2}}\left(k^{2}+\hat{\sigma}+\mu / \nu\right)\left(a_{k} \cos k x+b_{k} \sin k x\right)=0
\end{aligned}
$$

where $\hat{\sigma}=\sigma / \nu$. 
We further see that

$$
\begin{aligned}
J\left(\cos s x_{2}, \cos \left(k_{1} x_{1}+k_{2} x_{2}\right)\right) & =-k_{1} s \sin s x_{2} \sin \left(k_{1} x_{1}+k_{2} x_{2}\right)= \\
& \frac{k_{1} s}{2}\left(\cos \left(k_{1} x_{1}+\left(k_{2}+s\right) x_{2}\right)-\cos \left(k_{1} x_{1}+\left(k_{2}-s\right) x_{2}\right)\right), \\
J\left(\cos s x_{2}, \sin \left(k_{1} x_{1}+k_{2} x_{2}\right)\right) & =k_{1} s \sin s x_{2} \cos \left(k_{1} x_{1}+k_{2} x_{2}\right)= \\
& \frac{k_{1} s}{2}\left(\sin \left(k_{1} x_{1}+\left(k_{2}+s\right) x_{2}\right)-\sin \left(k_{1} x_{1}+\left(k_{2}-s\right) x_{2}\right)\right),
\end{aligned}
$$

and, as in [23], [28], we obtain the following recurrence relation for the coefficients $a_{k}$ (the equation for $b_{k}$ is exactly the same):

$$
\begin{array}{r}
-\left(\frac{k_{1}^{2}+\left(k_{2}+s\right)^{2}-s^{2}}{k_{1}^{2}+\left(k_{2}+s\right)^{2}}\right) a_{k_{1} k_{2}+s}+\left(\frac{k_{1}^{2}+\left(k_{2}-s\right)^{2}-s^{2}}{k_{1}^{2}+\left(k_{2}-s\right)^{2}}\right) a_{k_{1} k_{2}-s}+ \\
+\frac{2 \sqrt{2} \pi}{\lambda K k_{1}}\left(k_{1}^{2}+k_{2}^{2}+\hat{\sigma}+\mu / \nu\right) a_{k_{1} k_{2}}=0
\end{array}
$$

We set here

$$
a_{k_{1} k_{2}}\left(\frac{k^{2}-s^{2}}{k^{2}}\right)=: c_{k_{1} k_{2}}
$$

and

$$
\begin{array}{r}
k_{1}=t, \quad k_{2}=s n+r, \quad \text { and } \quad c_{t s n+r}=e_{n}, \\
t=1,2, \ldots, \quad r \in \mathbb{Z}, \quad r_{\min }<r<r_{\max }
\end{array}
$$

where the numbers $r_{\min }, r_{\max }$ satisfy $r_{\max }-r_{\min }<s$ and will be specified below, and obtain, for each $t$ and $r$, the following recurrence relation:

$$
d_{n} e_{n}+e_{n-1}-e_{n+1}=0, \quad n=0, \pm 1, \pm 2, \ldots
$$

where

$$
d_{n}=\frac{2 \sqrt{2} \pi\left(t^{2}+(s n+r)^{2}\right)\left(t^{2}+(s n+r)^{2}+\widetilde{\sigma}\right)}{\left(t^{2}+(s n+r)^{2}-s^{2}\right) \Lambda t}, \quad \tilde{\sigma}=\hat{\sigma}+\mu / \nu=\sigma / \nu+\mu / \nu
$$

and where we have set

$$
\Lambda=\lambda K=\lambda(s) K(s, \mu, \nu)=\lambda(s) \cdot \frac{s^{2}}{s^{2}+\mu / \nu} .
$$

We note that, for $\mu=0$, it follows from (2.28) that $\Lambda=\lambda$ and the recurrence relation $(2.26),(2.27)$ agrees exactly with the recurrence relation from [28].

We look for non-trivial decaying solutions $\left\{e_{n}\right\}$ of (2.26), (2.27). Each non-trivial decaying solution with 


$$
\operatorname{Re} \tilde{\sigma}>\frac{\mu}{\nu}
$$

produces an unstable eigenfunction $\varphi$ of the eigenvalue problem (2.23) with eigenvalue $\sigma$ satisfying

$$
\operatorname{Re} \sigma>0
$$

LEMMA 2.2. Given an integer $s>0$, let a pair of integers $(t, r)$ satisfying the conditions

$$
\begin{array}{r}
t^{2}+r^{2}<s^{2} / 3, \quad t^{2}+(-s+r)^{2}>s^{2}, \quad t^{2}+(s+r)^{2}>s^{2}, \quad t \geq \delta s, \\
r_{\min }<r<r_{\max }, \quad r_{\min }=-\frac{s}{6}, \quad r_{\max }=\frac{s}{6}, \quad 0<\delta<1 / \sqrt{3},
\end{array}
$$

be fixed. For every $\Lambda>0$, there exists a unique real eigenvalue $\widetilde{\sigma}=\widetilde{\sigma}(\Lambda)$ for which the recurrence relation $(2.26),(2.27)$ has a non-trivial decaying solution. Furthermore, $\tilde{\sigma}(\Lambda)$ increases monotonically as $\Lambda \rightarrow \infty$ and satisfies the inequality

$$
c_{1}(t, r, s) \Lambda \leq \widetilde{\sigma}(\Lambda) \leq c_{2}(t, r, s) \Lambda .
$$

The unique $\Lambda_{\mu / \nu}=\Lambda_{\mu / \nu}(s)$ solving the equation

$$
\widetilde{\sigma}\left(\Lambda_{\mu / \nu}\right)=\mu / \nu
$$

satisfies the inequality

$$
2 \pi \delta s<\Lambda_{\mu / \nu}(s)<4 \pi \sqrt{\frac{5}{6}} \delta^{-2} \cdot \frac{\left(\left(s^{2} / 3+\mu / \nu\right)\left(5 s^{2} / 3+\mu / \nu\right)\right)^{1 / 2}}{s} .
$$

Proof. The proof of this lemma is basically a repetition of the proof of Theorem 2.2 in [23], with $\lambda$ replaced by $\Lambda$ and $\hat{\sigma}$ by $\widetilde{\sigma}$ (and $\gamma=0$ ).

We see from [23] that the following inequalities hold for any $(t, r)$ satisfying (2.30):

$$
\begin{gathered}
s^{2}<t^{2}+(-s+r)^{2}<(5 / 3) s^{2}, \\
s^{2}<t^{2}+(s+r)^{2}<(5 / 3) s^{2} .
\end{gathered}
$$

Exactly as in [23], we prove estimate (2.31), which shows that, for $(t, r)$ satisfying (2.30), there exists a unique $\widetilde{\sigma}$ which increases monotonically with $\Lambda$ and, hence, $\tilde{\sigma}=\mu / \nu$ for some $\Lambda=\Lambda_{\mu / \nu}: \widetilde{\sigma}\left(\Lambda_{\mu / \nu}\right)=\mu / \nu$. To estimate $\Lambda_{\mu / \nu}$, we have, by monotonicity, $\Lambda_{\mu / \nu}>\Lambda_{0}$. Hence, we can use the lower bound for $\Lambda_{0}$ from [23], which gives the first inequality in (2.31).

In order to derive an upper bound for $\Lambda_{\mu / \nu}(s)$, we consider the case $r \geq 0$, the case $r<0$ being completely similar. If $r \geq 0$, we set $\widetilde{\sigma}=\mu / \nu$ in expression (54) in [23] and use inequality (57) in [23]. We obtain

$$
\Lambda_{\mu / \nu}<
$$

$\frac{4 \pi\left(t^{2}+r^{2}+\mu / \nu\right)^{1 / 2}\left(t^{2}+(s+r)^{2}+\mu / \nu\right)^{1 / 2}}{t}\left(\frac{t^{2}+r^{2}}{s^{2}-t^{2}-r^{2}}\right)^{1 / 2}\left(\frac{t^{2}+(s+r)^{2}}{t^{2}+(s+r)^{2}-s^{2}}\right)^{1 / 2}$. 
By (2.33), we see that $t^{2}+(s+r)^{2}<(5 / 3) s^{2}$. Next, since $r \geq 0$, we have $t^{2}+(s+r)^{2}-$ $s^{2} \geq \delta^{2} s^{2}$. Finally, since $t^{2}+r^{2}<s^{2} / 3$, we have $\left(t^{2}+r^{2}\right) /\left(s^{2}-t^{2}-r^{2}\right)<1 / 2$. Combining all these inequalities and the inequality $t \geq \delta s$, we obtain from (2.34) the second inequality in (2.32).

We denote by $A(\delta)$ the region in the $(t, r)$-plane satisfying (2.30). Denoting by $d(s)$ the number of points of the integer lattice inside the region $A(\delta)$, we obviously have

$$
d(s):=\#\left\{(t, r) \in D(s)=\mathbb{Z}^{2} \cap A(\delta)\right\} \simeq a(\delta) \cdot s^{2} \quad \text { as } \quad s \rightarrow \infty,
$$

where $a(\delta) \cdot s^{2}=|A(\delta)|$ is the area of the region $A(\delta)$. Next, taking into account that the analysis of the recurrence relation for the coefficients $b_{k_{1} k_{2}}$ is exactly the same, we see by Lemma 2.2 that, for each pair $(t, r) \in D(s)$ (equivalently, for each $(t, r)$ satisfying (2.30)) and parameter $\Lambda$ (see (2.28)) chosen as follows:

$$
\Lambda=\Lambda_{\mu / \nu}=4 \pi \sqrt{\frac{5}{6}} \delta^{-2} \cdot \frac{\left(\left(s^{2} / 3+\mu / \nu\right)\left(5 s^{2} / 3+\mu / \nu\right)\right)^{1 / 2}}{s},
$$

there exists a unique real positive eigenvalue $\widetilde{\sigma}>\mu / \nu$ of multiplicity two. Hence, there exists a positive eigenvalue $\sigma>0$ of the original eigenvalue problem (2.23) of multiplicity two. Therefore, the dimension of the unstable manifold near the stationary solution $\varphi_{s}$ is at least $2 d(s)$ and we obtain as a result

$$
\operatorname{dim} \mathcal{A} \geq 2 d(s) \simeq 2 a(\delta) \cdot s^{2} .
$$

The parameter $s$ was arbitrary so far. We now set

$$
s^{2}=\frac{\mu}{\nu}=\frac{\mu L}{\nu} .
$$

(To be completely rigorous, we have to make sure that $\mu / \nu$ is a complete square, but, anyway, we already have the " $\_$" sign in (2.37).) Therefore,

$$
\operatorname{dim} \mathcal{A} \gtrsim 2 a(\delta) \frac{\mu}{\nu} .
$$

We now recall the definition of $\Lambda(2.28)$ :

$$
\Lambda=\lambda(s) \cdot \frac{s^{2}}{s^{2}+\mu / \nu} .
$$

Setting $s^{2}=\mu / \nu$ here and in (2.36), we obtain the equation for $\lambda$, which gives

$$
\lambda(s)=\lambda\left((\mu / \nu)^{1 / 2}\right)=\frac{32 \pi}{3} \sqrt{\frac{5}{3}} \delta^{-2}\left(\frac{\mu}{\nu}\right)^{1 / 2} .
$$

We compute the numbers

$$
G_{1}=\frac{\|\operatorname{rot} f\| L}{\nu \mu} \quad \text { and } \quad G_{2}=\frac{\|\operatorname{rot} f\|^{2}}{\nu \mu^{3}},
$$

for $f=f_{s}$ and $s=(\mu / \nu)^{1 / 2}$. In view of (2.19) and (2.39), we have 


$$
\left\|\operatorname{rot} f_{s}\right\|=\nu^{2} \lambda(s) s^{3}=\frac{32 \pi}{3} \sqrt{\frac{5}{3}} \delta^{-2} \mu^{2} .
$$

Hence, we find that

$$
G_{1}=\frac{32 \pi}{3} \sqrt{\frac{5}{3}} \delta^{-2} \frac{\mu}{\nu} \quad \text { and } \quad G_{2}=\frac{5120 \pi^{2}}{27} \delta^{-4} \frac{\mu}{\nu} .
$$

Note that, for the Kolmogorov forcing (2.17) and $s=(\mu / \nu)^{1 / 2}$, the numbers $G_{1}$ and $G_{2}$ are of the same order with respect to $s$. Expressing estimate (2.38) in terms of the numbers $G_{1}$ and $G_{2}(2.41)$ and optimizing with respect to $\delta \in(0,1 / \sqrt{3})$, we obtain

$$
\begin{aligned}
\operatorname{dim}_{F} \mathcal{A} & \gtrsim \frac{3}{16 \pi} \sqrt{\frac{3}{5}} \max _{0<\delta<1 / \sqrt{3}}\left(a(\delta) \delta^{2}\right) G_{1}=3.2 \ldots \cdot 10^{-4} G_{1}, \\
\operatorname{dim}_{F} \mathcal{A} & \gtrsim \frac{27}{2560 \pi^{2}} \max _{0<\delta<1 / \sqrt{3}}\left(a(\delta) \delta^{4}\right) G_{2}=1.5 \ldots \cdot 10^{-6} G_{2},
\end{aligned}
$$

where $\max _{0<\delta<1 / \sqrt{3}}\left(a(\delta) \delta^{2}\right)=6.93 \cdot 10^{-3}, \max _{0<\delta<1 / \sqrt{3}}\left(a(\delta) \delta^{4}\right)=1.4 \cdot 10^{-3}$ and $a(\delta)$ is defined in (2.35).

Combining these results and Theorem 2.1, we have proved the following theorem.

TheOREM 2.3. The dimension of the global attractor $\mathcal{A}$ for equation (1.3) with the Kolmogorov forcing (2.17) satisfies the following sharp two-sided estimates:

$$
\begin{gathered}
3.2 \cdot 10^{-4} \frac{\|\operatorname{rot} f\| L}{\nu \mu} \lesssim \operatorname{dim}_{F} \mathcal{A} \leq 2.8 \frac{\|\operatorname{rot} f\| L}{\nu \mu}, \\
1.5 \cdot 10^{-6} \frac{\|\operatorname{rot} f\|^{2}}{\nu \mu^{3}} \lesssim \operatorname{dim}_{F} \mathcal{A} \leq 0.48 \frac{\|\operatorname{rot} f\|^{2}}{\nu \mu^{3}} .
\end{gathered}
$$

Remark 1. Inequalities (2.43) are not contradictory in the sense that the lower bound in one inequality exceeds the upper bound in the other. In fact, the two lower bounds in (2.43) are the same and are merely estimate (2.38) written in two different ways.

Remark 2. Our lower bounds can be reformulated as follows. Let $\mu>0$ be arbitrary but fixed. Then, for any $\nu>0$ (such that $\mu /\left(\nu L^{2}\right)>1$ ), there exists a (Kolmogorov) right-hand side $f$ with norm $\|\operatorname{rot} f\|$ independent of $\nu$ and depending only on $\mu$ such that the following estimate holds:

$$
\operatorname{dim}_{F} \mathcal{A} \geq C_{1} \frac{\|\operatorname{rot} f\| L}{\nu \mu}=\frac{\operatorname{const}(\mu)}{\nu} .
$$

Remark 3. Taking $l=0$ allows to simplify significantly the derivation of the lower bounds. As seen above, the Coriolis term makes no difference in our estimates of the dimension of the global attractor since it is anti-symmetric. It will, however, make a difference in the dynamical structure of the attractor and bifurcations.

Remark 4. Similar results have been established in [18], [19]. The estimates reported in this work are much sharper. 


\section{Equations on the sphere and in a bounded domain}

We now consider the Navier-Stokes perturbation of the damped Euler equations on the sphere and in a bounded domain.

3.1. Equations on the sphere. Let $S^{2}=S_{R}^{2}$ be the sphere of radius $R$ with spherical coordinates $\lambda, 0 \leq \lambda \leq 2 \pi$ (the longitude), and $\phi,-\pi / 2 \leq \phi \leq \pi / 2$ (the geographical latitude). Suppose that the sphere rotates around the axis through the poles $\phi= \pm \pi / 2$ with constant angular velocity $\omega$. Then the Navier-Stokes system with damping reads

$$
\begin{array}{r}
\partial_{t} u+\nabla_{u} u+n l \times u=\nu \Delta u-\mu u-\nabla p+f, \\
\operatorname{div} u=0, \quad u(0)=u_{0} .
\end{array}
$$

Here, $u$ is the tangent velocity vector, $p$ is the pressure, $n$ is the outward unit normal vector, $n l \times u$ is the Coriolis acceleration ( $l=2 \omega \sin \phi$ is the Coriolis parameter), $\nabla_{u} u$ is the covariant derivative of $u$ along $u$ with respect to the metric of the sphere for which we have the following global representation:

$$
\nabla_{u} u=\nabla \frac{u^{2}}{2}-u \times \operatorname{rot} u
$$

The operators div and $\nabla=\operatorname{grad}$ have the conventional meaning: for a scalar $\psi$ and a vector $u=u^{\lambda} e_{\lambda}+u^{\phi} e_{\phi}$ with physical components (that is, $|u|^{2}=\left(u^{\lambda}\right)^{2}+\left(u^{\phi}\right)^{2}$ ), we have

$$
\nabla \psi=\frac{1}{R \cos \phi} \partial_{\lambda} \psi \cdot e_{\lambda}+\frac{1}{R} \partial_{\phi} \psi \cdot e_{\phi}, \quad \operatorname{div} u=\frac{1}{R \cos \phi}\left(\partial_{\lambda} u^{\lambda}+\partial_{\phi}\left(\cos \phi u^{\phi}\right)\right) .
$$

Next, the operator rot acts on tangent vectors $u$ and scalars $\psi$ (which are identified with normal vectors) as follows [22]:

$$
\operatorname{rot} u=-n \cdot \operatorname{div}(n \times u), \quad \operatorname{rot} \psi=-n \times \nabla \psi .
$$

Finally, $\Delta u$ is the vector Laplacian of $u$ (the Laplace-de Rham operator)

$$
\Delta u=\nabla \operatorname{div} u-\operatorname{rot}(\operatorname{rot} u)=\nabla \operatorname{div} u-n \times \nabla \operatorname{div}(n \times u) .
$$

As in the space-periodic case, we denote by $P$ the orthogonal projection in $L_{2}\left(T S^{2}\right)$ onto $H$ which is the closed subspace of solenoidal vector fields. Applying $P$ to $(3.1)$, we obtain

$$
\partial_{t} u+B(u, u)+\nu A u+P(n l \times u)=-\mu u+f, \quad u(0)=u_{0},
$$

where

$$
\begin{array}{r}
B(u, u)=P\left(\nabla_{u} u\right)=-P(u \times \operatorname{rot} u), B(u, v)=P\left(\nabla_{u} v\right), \\
(B(u, v), w)=b(u, v, w)=\int_{S^{2}} \nabla_{u} v(s) \cdot w(s) d S \quad \text { and } \quad A u=\operatorname{rot}(\operatorname{rot} u) .
\end{array}
$$

Furthermore, we have orthogonality relations and formulas for the integration by parts [22] that are totally similar to the space-periodic case:

$$
b(u, v, v)=0, \quad b(v, v, u)=-b(v, u, v), \quad b(u, u, A u)=0, \quad(n l \times u, A u)=0,
$$


and

$$
(A u, v)=(\operatorname{rot} u, \operatorname{rot} v) .
$$

Now, the existence of the semigroup $S_{t}: H \rightarrow H$ and the global attractor $\mathcal{A} \Subset H$ is established as in the space-periodic case [22].

Taking the scalar product of (3.2) with $A u$ and integrating by parts using (3.3) and (3.4), we obtain, as above, that the following inequality holds on the attractor $\mathcal{A}$ :

$$
\|\operatorname{rot} u(t)\| \leq \frac{\|\operatorname{rot} f\|}{\mu} .
$$

THEOREM 3.1. The fractal dimension of $\mathcal{A}$ satisfies the following upper bound:

$$
\operatorname{dim}_{F} \mathcal{A} \leq \min \left(2 \frac{\|\operatorname{rot} f\|}{\nu \mu \lambda_{1}^{1 / 2}}, \frac{1}{2} \frac{\|\operatorname{rot} f\|^{2}}{\nu \mu^{3}}\right)=\min \left(\sqrt{2} \frac{\|\operatorname{rot} f\| R}{\nu \mu}, \frac{1}{2} \frac{\|\operatorname{rot} f\|^{2}}{\nu \mu^{3}}\right) .
$$

Proof. The proof is similar to the space-periodic case and will be only outlined. We consider the linearized equation

$$
\partial_{t} U=-\nu A U-\mu U-B(U, u(t))-B(u(t), U)-P(n l \times U)=: \mathcal{L}\left(t, u_{0}\right) U,
$$

and estimate the $m$-trace of the operator $\mathcal{L}$. As in section 1 , we use $(3.3),(3.4)$ to find that

$$
\begin{aligned}
& \operatorname{Tr}\left[\mathcal{L}\left(t, u_{0}\right) \circ Q_{m}(t)\right]=\sum_{j=1}^{m}\left(\mathcal{L}\left(t, u_{0}\right) v_{j}, v_{j}\right)= \\
& -\nu \sum_{j=1}^{m}\left\|\operatorname{rot} v_{j}\right\|^{2}-\mu m+\sum_{j=1}^{m} b\left(v_{j}, u(t), v_{j}\right) \leq \\
& -\nu \sum_{j=1}^{m}\left\|\operatorname{rot} v_{j}\right\|^{2}-\mu m+2^{-1 / 2}\|\rho\|\|\operatorname{rot} u(t)\|,
\end{aligned}
$$

where $\rho(x)=\sum_{j=1}^{m}\left|v_{j}(x)\right|^{2}$ and where, instead of (2.6), we have used estimate (3.9) in Lemma 3.2 below. The spectrum of the Laplacian on the sphere is well-known. Hence,

$$
\sum_{j=1}^{m}\left\|\operatorname{rot} v_{j}\right\|^{2} \geq \sum_{j=1}^{m} \lambda_{j} \geq \lambda_{1} c_{\mathrm{S}^{2}-\mathrm{spec}} m^{2}
$$

where (see [23])

$$
c_{\mathrm{S}^{2}-\mathrm{spec}}=\frac{1}{4} .
$$

Concerning the Lieb-Thirring inequality on the sphere, we have

$$
\|\rho\|^{2}=\int_{S^{2}}\left(\sum_{j=1}^{m}\left|v_{j}(s)\right|^{2}\right)^{2} d S \leq c_{\mathrm{S}^{2}-\mathrm{LT}} \sum_{j=1}^{m}\left\|\operatorname{rot} v_{j}\right\|^{2}
$$


where we can take the following explicit upper estimate for $c_{\mathrm{S}^{2}-\mathrm{LT}}$ (see [20]):

$$
c_{\mathrm{S}^{2}-\mathrm{LT}} \leq 2 .
$$

We can now complete the proof of the theorem as in section 1 and obtain (3.5); the equality in (3.5) following from the fact that $\lambda_{1}=2 R^{-2}$.

Lemma 3.2. If $\operatorname{div} u=0$ and $\left\{v_{j}\right\}_{j=1}^{m}, u \in H_{1}$, then

$$
\sum_{j=1}^{m} b\left(v_{j}, u, v_{j}\right)=\sum_{j=1}^{m} \int_{S^{2}}\left(\nabla_{v_{j}} u(s) \cdot v_{j}(s)\right) d S \leq 2^{-1 / 2}\|\rho\|\|\operatorname{rot} u\| .
$$

Proof. Let a point $s \in S^{2}$ be arbitrary but fixed. We first show that the following pointwise inequality holds at $s$ :

$$
\nabla_{v} u(s) \cdot v(s) \leq 2^{-1 / 2}|v(s)|^{2}|\nabla u(s)|,
$$

where

$$
(\nabla u(s))^{2}=\sum_{i, j=1}^{2}\left(\nabla_{i} u^{j}(s)\right)^{2}
$$

and

$$
\nabla_{k} u^{i}=\frac{\partial u^{i}}{\partial x_{k}}+\Gamma_{j k}^{i} u^{j}, \quad\left(\nabla_{v} u\right)^{i}=v^{k} \nabla_{k} u^{i}, \quad \nabla_{v} u \cdot v=v^{k} \nabla_{k} u^{i} v^{j} g_{i j}=v^{k} \nabla_{k} u^{i} v_{i} .
$$

Here, $x_{k}$ denote the local coordinates near $s, g_{i j}$ is the metric, and $\Gamma_{j k}^{i}$ are the corresponding Christoffel symbols. The vector field $u$ has contravariant components $u^{i}, i=1,2$. As usual, the summation convention is assumed. We now choose the local coordinates $x_{k}$ so that, at $s, g_{i j}=\delta_{i j}$. It suffices to use spherical coordinates chosen so that the equator $(\phi=0)$ passes through $s$. Then, at $s$, we have $v_{i}=g_{i j} v^{j}=v^{i}$ and, hence, $|v|^{2}=\left(v^{1}\right)^{2}+\left(v^{2}\right)^{2}$. $B$ :

Next, we define, at $s$ (in the linear algebraic sense), the vector $a$ and the matrix

$$
a_{i}=v^{i}=v_{i}, \quad B=\left(\begin{array}{cc}
\nabla_{1} u^{1} & \nabla_{1} u^{2} \\
\nabla_{2} u^{1} & \nabla_{2} u^{2}
\end{array}\right) .
$$

Then, at $s$, we have (see [8], Lemma 4.1)

$$
\nabla_{v} u \cdot u=B a \cdot a=\frac{1}{2}\left(B+B^{*}\right) a \cdot a \leq|\lambda||a|^{2},
$$

where $\lambda$ is the largest (in absolute value) eigenvalue of the symmetric matrix $\frac{1}{2}(B+$ $\left.B^{*}\right)$. Since $\operatorname{div} u=\nabla_{i} u_{i}=0$, it follows that the trace of $\frac{1}{2}\left(B+B^{*}\right)$ vanishes and the eigenvalues are $\lambda>0$ and $-\lambda$, where, using the characteristic polynomial, we have

$$
\lambda^{2}=\left(\nabla_{1} u^{1}\right)^{2}+\frac{1}{4}\left(\nabla_{1} u^{2}+\nabla_{2} u^{1}\right)^{2} \leq \frac{1}{2}\left(\sum_{i, j=1}^{2}\left(\nabla_{i} u(s)^{j}\right)^{2}\right)=\frac{1}{2}|\nabla u(s)|^{2} .
$$

Since, in (3.11), $|a(s)|^{2}=|v(s)|^{2}$, this proves (3.10) for an arbitrary point $s \in S^{2}$. 
There remains to integrate (3.10):

$$
\begin{aligned}
& \sum_{j=1}^{m} b\left(v_{j}, u, v_{j}\right)=\int_{S^{2}} \sum_{j=1}^{m} \nabla_{v_{j}} u(s) \cdot v_{j}(s) d S \leq 2^{-1 / 2} \int_{S^{2}} \sum_{j=1}^{m}\left|v_{j}(s)\right|^{2}|\nabla u(s)| d S= \\
& 2^{-1 / 2} \int_{S^{2}} \rho(s)|\nabla u(s)| d S \leq 2^{-1 / 2}\|\rho\|\|\nabla u\| \leq 2^{-1 / 2}\|\rho\|\|\operatorname{rot} u\|,
\end{aligned}
$$

where we have used the inequality

$$
\|\nabla u\| \leq\|\operatorname{rot} u\| .
$$

To prove (3.12), we use the following identity (a variant of the Weitzenböck formula, see [22], Remark 4.3):

$$
\Delta u=\nabla \operatorname{div} u-\operatorname{rot}(\operatorname{rot} u)=\nabla^{2} u-u,
$$

where $\left(\nabla^{2} u\right)^{i}=g^{k l} \nabla_{k} \nabla_{l} u^{i}$. Taking the scalar product with $-u$ and integrating by parts, using the formulas

$$
(\operatorname{rot}(\operatorname{rot} u), u)=\|\operatorname{rot} u\|^{2}, \quad-\left(\nabla^{2} u, u\right)=\|\nabla u\|^{2},
$$

we obtain the identity $\|\nabla u\|^{2}+\|u\|^{2}=\|\operatorname{rot} u\|^{2}$. Hence,

$$
\|\nabla u\|<\|\operatorname{rot} u\|, \quad u \neq 0
$$

which proves (3.12) and the lemma. We finally observe that the orthonormality of the $v_{j}$ s does not play a role in this lemma.

Setting $m=1$, we obtain the following explicit estimate for the form $b$.

COROLlary 3.3. The following estimate holds for $u, v \in H_{1} \cap H$ :

$$
|b(v, v, u)|=|b(v, u, v)| \leq c\|v\|\|\operatorname{rot} v\|\|\operatorname{rot} u\|,
$$

where $c \leq 1$.

Proof. We set $m=1$ and $v_{1}=v=\tilde{v} /\|\tilde{v}\|$ in the Lieb-Thirring inequality (3.7). We obtain (omitting the tilde sign) the Ladyzhenskaya-Gagliardo-Nirenberg inequality for solenoidal vector fields on the sphere:

$$
\|v\|_{L_{4}}^{4} \leq c_{\mathrm{S}^{2}-\mathrm{LGN}}\|v\|^{2}\|\operatorname{rot} v\|^{2}
$$

where $c_{\mathrm{S}^{2}-\mathrm{LGN}} \leq c_{\mathrm{S}^{2}-\mathrm{LT}} \leq 2$. Setting $m=1$ in $(3.9)$, we find

$$
|b(v, u, v)| \leq 2^{-1 / 2}\|v\|_{L_{4}}^{2}\|\operatorname{rot} u\| \leq\left(\frac{c_{\mathrm{S}^{2}-\mathrm{LGN}}}{2}\right)^{1 / 2}\|v\|\|\operatorname{rot} v\|\|\operatorname{rot} u\|,
$$

which proves the lemma.

3.2. Equations in a bounded domain. Let $\Omega \subset \mathbb{R}^{2}$ be a bounded domain with a boundary of class $C^{2}$. Let $n$ be the outward unit normal vector. We further assume, for simplicity, that $\Omega$ is simply connected. We consider our Navier-Stokes system with damping supplemented with the so-called free boundary conditions:

$$
\begin{gathered}
\partial_{t} u+\sum_{i=1}^{2} u^{i} \partial_{i} u=-\mu u+\nu \Delta u-\nabla p+f \\
\operatorname{div} u=0 \\
\left.u \cdot n\right|_{\partial \Omega}=0,\left.\quad \operatorname{rot} u\right|_{\partial \Omega}=0 .
\end{gathered}
$$


We introduce the phase space $H$ :

$$
H=\left\{u \in L_{2}(\Omega)^{2} ; \operatorname{div} u=0,\left.u \cdot n\right|_{\partial \Omega}=0\right\} .
$$

Since $\Omega$ is simply connected, we also have (see [34], Appendix 1)

$$
H=\left\{u=\operatorname{rot} \psi, \psi \in H_{0}^{1}(\Omega)\right\} .
$$

In other words, a vector field from $H$ has a unique single valued stream function $\psi$ vanishing at the boundary of $\Omega$.

Let $P$ be the orthogonal projection, $P: L_{2}(\Omega)^{2} \rightarrow H$. Applying $P$ to (3.15), we write (3.15) as an evolution equation of the form (2.2):

$$
\partial_{t} u+B(u, u)+\nu A u=-\mu u+f, \quad u(0)=u_{0},
$$

where $B(u, v)=P\left(\sum_{i=1}^{2} u^{i} \partial_{i} v\right)$ and $A=-P \Delta$ is the Stokes operator with domain

$$
D(A)=\left\{u \in H^{2}(\Omega)^{2} ; \operatorname{div} u=0,\left.u \cdot n\right|_{\partial \Omega}=0,\left.\operatorname{rot} u\right|_{\partial \Omega}=0\right\} .
$$

As above, the following orthogonality relation is essential:

$$
(B(u, u), A u)=0, \quad u \in D(A) .
$$

Next, as in the case of a torus and a sphere, the spectrum $\left\{\lambda_{k}\right\}_{k=1}^{\infty}$ of the Stokes operator coincides with the spectrum of the scalar Dirichlet problem $-\Delta \psi_{k}=\lambda_{k} \psi_{k}$, $\left.\psi_{k}\right|_{\partial \Omega}=0$. Hence,

$$
\sum_{j=1}^{m} \lambda_{j} \geq \lambda_{1} c_{\Omega-\mathrm{spec}} m^{2}
$$

As above, the Lieb-Thirring inequality is essential.

LEMma 3.4. Suppose that the family $\left\{v_{j}\right\}_{j=1}^{m} \in H^{1}(\Omega)^{2} \cap H$ is orthonormal: $\int_{\Omega} v_{i}(x)$. $v_{j}(x) d x=\delta_{i j}$.

Then the following inequality holds

$$
\int_{\Omega} \rho(x)^{2} d x \leq c_{\Omega-\mathrm{LT}} \sum_{j=1}^{m}\left\|\operatorname{rot} v_{j}\right\|^{2},
$$

where $\rho(x)=\sum_{j=1}^{m} v_{j}(x)^{2}$ and $c_{\Omega-\mathrm{LT}}$ is a dimensionless constant depending on the shape of $\Omega$ only: $c_{\lambda \Omega-\mathrm{LT}}=c_{\Omega-\mathrm{LT}}, \lambda>0$.

Proof. We infer from the generalized Lieb-Thirring inequality (see Theorem 4.1 in the Appendix in [35]) that there exists dimensionless constants $k_{1}$ and $k_{2}$ depending on the shape of $\Omega$ only such that

$$
\int_{\Omega} \rho(x)^{2} d x \leq k_{1} \sum_{j=1}^{m}\left\|\nabla v_{j}\right\|^{2}+\frac{k_{2}}{|\Omega|} \int_{\Omega} \rho(x) d x .
$$

Since $\Omega$ is simply connected, we have the Poincaré inequality

$$
\|v\|^{2} \leq \lambda_{1}^{-1}\|\operatorname{rot} v\|^{2}
$$


and, hence, the second term in the right-hand side of (3.19) is bounded by the righthand side of (3.18). To complete the proof, it suffices to prove the inequality

$$
\|\nabla v\|^{2} \leq k_{3}\|\operatorname{rot} v\|^{2} .
$$

Introducing the stream function $\psi \in H^{2} \cap H_{0}^{1}, v=\left\{-\partial_{2} \psi, \partial_{1} \psi\right\}$, we see that this inequality is equivalent to the inequality

$$
\sum_{i, j=1}^{2}\left\|\partial_{i j}^{2} \psi\right\|^{2} \leq k_{3}\|\Delta \psi\|^{2}
$$

which is well-known in the elliptic theory.

Having done this preliminary work, we can proceed as in section 1 and obtain the following result.

TheOREM 3.5. Equation (3.15) has a global attractor $\mathcal{A}$ whose fractal dimension satisfies the following upper bound:

$$
\operatorname{dim}_{F} \mathcal{A} \leq \min \left(c^{\prime} \frac{\|\operatorname{rot} f\|}{\nu \mu \lambda_{1}^{1 / 2}}, c^{\prime \prime} \frac{\|\operatorname{rot} f\|^{2}}{\nu \mu^{3}}\right), \text { where } c^{\prime}=\left(\frac{c_{\Omega-\mathrm{LT}}}{2 c_{\Omega-\mathrm{spec}}}\right)^{1 / 2}, c^{\prime \prime}=\frac{c_{\Omega-\mathrm{LT}}}{4}
$$

Appendix A. Lieb-Thirring inequalities for space-periodic solenoidal vector functions.

Inequality (2.9) follows from the generalized Lieb-Thirring inequality in [35]. The aim of this section is to prove the explicit estimate of the constant (2.10).

We first consider the scalar case. We basically follow the strategy of the proof of the general result in [35], paying special attention to explicit expressions of the constants involved. We assume that $L=1$ so that $T^{2}=[0,2 \pi]^{2}$. This involves no loss of generality since the constants in the Lieb-Thirring inequalities are scale invariant and depend only on the aspect ratio of the torus. Let $\Pi$ be the orthogonal projection in $L_{2}\left(T^{2}\right)$ onto the space of functions with mean value zero:

$$
\Pi h=h-\frac{1}{4 \pi^{2}} \int_{T^{2}} h(x) d x .
$$

We set

$$
H=\Pi L_{2}\left(T^{2}\right), \quad H_{1}=H^{1}\left(T^{2}\right) \cap H .
$$

We order the eigenvalues of $-\Delta$ in $H$ according to magnitude and multiplicity:

$$
1=\lambda_{1} \leq \lambda_{2} \leq \ldots, \quad\left\{\lambda_{j}, j=1, \ldots\right\}=\left\{k^{2}=k_{1}^{2}+k_{2}^{2}, k=\left(k_{1}, k_{2}\right) \in \mathbb{Z}_{0}^{2}\right\},
$$

where $\mathbb{Z}_{0}^{2}=\mathbb{Z}^{2} \backslash\{0\}$. The corresponding basis of orthonormal eigenfunctions $w_{j}(x)$, $-\Delta w_{j}=\lambda_{j} w_{j}$, is the basis of trigonometric functions

$$
\begin{array}{r}
\bigcup_{j \in \mathbb{N}} w_{j}(x)=\bigcup_{k \in \mathbb{Z}_{+}^{2}}\left\{(\sqrt{2} \pi)^{-1} \sin k x, \quad(\sqrt{2} \pi)^{-1} \cos k x\right\}, \\
\mathbb{Z}_{+}^{2}=\left\{k \in \mathbb{Z}_{0}^{2}, \quad k_{1} \geq 0, \quad k_{2} \geq 0\right\} \cup\left\{k \in \mathbb{Z}_{0}^{2}, \quad k_{1} \geq 1, k_{2} \leq 0\right\} .
\end{array}
$$


Similarly to (A.1), we write

$$
1=\Lambda_{1} \leq \Lambda_{2} \leq \ldots, \quad\left\{\Lambda_{j}, j=1, \ldots\right\}=\left\{k^{2}, k \in \mathbb{Z}_{+}^{2}\right\}
$$

and observe that

$$
\bigcup_{j=1}^{\infty}\left\{\lambda_{j}\right\}=\bigcup_{l=1}^{\infty}\left\{\Lambda_{l}, \Lambda_{l}\right\} .
$$

Hence, for $j \geq 1$, we have $\Lambda_{j}=\lambda_{2 j}=\lambda_{2 j-1}$ and, corresponding to each $\Lambda=\Lambda_{j}$, there are two eigenfunctions $u_{j}(x)=(\sqrt{2} \pi)^{-1} \sin k x$ and $v_{j}(x)=(\sqrt{2} \pi)^{-1} \cos k x$ for some uniquely defined $k=k(j)$. We obviously have

$$
u_{j}(x)^{2}+v_{j}(x)^{2}=\frac{1}{2 \pi^{2}}=: c_{2} .
$$

Next, we have the following lower bound for $\lambda_{j}$.

Proposition A.1. The following inequalities hold for $j \geq 1$ :

$$
\lambda_{j} \geq c_{1} j, \quad \Lambda_{j} \geq 2 c_{1} j, \quad \text { where } \quad c_{1}=\frac{1}{4} .
$$

Proof. We have the Weyl asymptotics for $\lambda_{j}$ (which can easily be proved in this particular case): $\lim _{j \rightarrow \infty} \lambda_{j} / j=1 / \pi>1 / 4$ and, therefore, $\lambda_{j}>j / 4$ for $j \geq j_{0}$. By a direct calculation, we find that $\lambda_{4}=1=4 / 4$ and $\lambda_{20}=5=20 / 4$. Hence, we have to verify that $\lambda_{j} \geq j / 4$ for finitely many $j<j_{0}$. We omit the details.

Finally, the second inequality in (A.5) follows from the fact that $\Lambda_{j}=\lambda_{2 j}$. $\square$

After these simple preliminaries, we consider, for a potential $f \in L_{2}\left(T^{2}\right)$, the quadratic form

$$
Q_{f}(h)=\|\nabla h\|^{2}+\int_{T^{2}} f(x) h^{2}(x) d x, \quad h \in H_{1},
$$

which is bounded from below, and the numbers

$$
\eta_{j}(f)=\max _{\psi_{1}, \ldots, \psi_{j-1} \in H_{1}} \min _{\substack{\left(\varphi, \psi_{i}\right)=0, i=1, \ldots, j-1 \\ \varphi \in H_{1},\|\varphi\|=1,}} Q_{f}(h) .
$$

The quadratic form (A.6) defines the Schrödinger operator

$$
-\Delta h+\Pi(f \Pi h),
$$

and the numbers $\eta_{j}$ are the eigenvalues of this operator.

Our aim is to estimate the negative trace of the operator (A.8)

$$
\sum_{\eta_{j} \leq 0}\left|\eta_{j}\right| \leq \mathrm{L}_{1}\left(T^{2}\right) \int_{T^{2}} f_{-}^{2}(x) d x,
$$

and, more precisely, to estimate the corresponding constant $\mathrm{L}_{1}\left(T^{2}\right)$ (since inequality (A.9) follows from a general theorem in [35]). Here,

$$
f_{-}(x)=\left\{\begin{array}{cc}
-f(x) & \text { for } \quad f(x) \leq 0 \\
0 & \text { for } \quad f(x)>0
\end{array}\right.
$$


THEOREM A.2. The constant $\mathrm{L}_{1}\left(T^{2}\right)$ satisfies the estimate

$$
\mathrm{L}_{1}\left(T^{2}\right) \leq \frac{3}{2 \pi}=0.478 \ldots \quad\left(\mathrm{L}_{1}\left(T^{2}\right) \leq 0.459 \ldots\right)
$$

Proof. We denote by $N_{r}(f)$ the number of eigenvalues $\eta_{j}(f)$ such that $\eta_{j}(f) \leq r$ :

$$
N_{r}(f)=\#\left\{\eta_{j}(f), \eta_{j}(f) \leq r\right\}
$$

and obtain

$$
\sum_{\eta_{j} \leq 0}\left|\eta_{j}\right|=\int_{0}^{\infty} N_{-r}(f) d r
$$

Using the Birman-Schwinger kernel [27], [35] and the convexity inequality of Lieb and Thirring [1], [27], we obtain

$N_{-r}(f) \leq \operatorname{Tr}(f+(1-t) r)_{-}^{k / 2}(\Pi(-\Delta+t r) \Pi)^{-k}(f+(1-t) r)_{-}^{k / 2}, \quad r>0, k \geq 1, t \in[0,1]$.

We first show that, for $k>1$,

$$
N_{-r}(f) \leq \frac{1}{\pi^{2}} B(1, k-1)(t r)^{1-k} \int_{T^{2}}(f(x)+(1-t) r)^{k} d x,
$$

where $B$ is the Beta function: $B(x, y)=\int_{0}^{1} t^{x-1}(1-t)^{y-1} d t=\Gamma(x) \Gamma(y) / \Gamma(x+y)$.

In fact, setting $g=(f+(1-t) r)_{-}$and using the basis of eigenfunctions, we have

$$
\left[g^{k / 2}(\Pi(-\Delta+t r) \Pi)^{-k} g^{k / 2} \varphi\right](x)=\int_{T^{2}} G\left(x, x^{\prime}\right) \varphi\left(x^{\prime}\right) d x^{\prime},
$$

where

$$
G\left(x, x^{\prime}\right)=\sum_{j=1}^{\infty}\left(\lambda_{j}+t r\right)^{-k} g(x)^{k / 2} w_{j}(x) g\left(x^{\prime}\right)^{k / 2} w_{j}\left(x^{\prime}\right)
$$

Hence,

$$
\begin{aligned}
& \operatorname{Tr} g^{k / 2}(\Pi(-\Delta+t r) \Pi)^{-k} g^{k / 2}=\int_{T^{2}} G(x, x) d x=\int_{T^{2}} \sum_{j=1}^{\infty}\left(\lambda_{j}+t r\right)^{-k} g(x)^{k} w_{j}(x)^{2} d x= \\
& \int_{T^{2}} \sum_{j=1}^{\infty}\left(\Lambda_{j}+t r\right)^{-k} g(x)^{k}\left(u_{j}(x)^{2}+v_{j}(x)^{2}\right) d x=c_{2} \sum_{j=1}^{\infty}\left(\Lambda_{j}+t r\right)^{-k} \int_{T^{2}} g(x)^{k} d x,
\end{aligned}
$$

which proves (A.11), since

$$
\sum_{j=1}^{\infty}\left(\Lambda_{j}+t r\right)^{-k} \leq \sum_{j=1}^{\infty}\left(2 c_{1} j+t r\right)^{-k} \leq \int_{0}^{\infty} \frac{d s}{\left(2 c_{1} s+t r\right)^{k}}=\frac{1}{2 c_{1}} B(1, k-1)(t r)^{1-k}
$$

and, by (A.4), (A.5), we have $c_{2} /\left(2 c_{1}\right)=1 / \pi^{2}$. 
Next, restricting $k$ to $k \in(1,2)$, we have

$$
\begin{aligned}
& \sum_{\eta_{j} \leq 0}\left|\eta_{j}\right|=\int_{0}^{\infty} N_{-r}(f) d r \leq \frac{1}{\pi^{2}} B(1, k-1) \int_{0}^{\infty} \int_{T^{2}}(t r)^{1-k}(f(x)+(1-t) r)_{-}^{k} d x d r= \\
& \frac{1}{\pi^{2}} B(1, k-1) \int_{T^{2}} \int_{0}^{\infty}(t r)^{1-k}(f(x)+(1-t) r)_{-}^{k} d r d x .
\end{aligned}
$$

We evaluate the inner integral by setting, for almost every $x$,

$$
r=\frac{1}{1-t} f_{-}(x) \rho .
$$

If $f \leq 0$ and $f_{-}=-f$, then $(f+(1-t) r)_{-}=f_{-}(\rho-1)_{-}$and

$$
\begin{aligned}
& \int_{0}^{\infty}(t r)^{1-k}(f(x)+(1-t) r)_{-}^{k} d r=t^{1-k}(1-t)^{k-2} f_{-}(x)^{2} \int_{0}^{\infty} \rho^{1-k}(\rho-1)_{-}^{k} d \rho= \\
& t^{1-k}(1-t)^{k-2} f_{-}(x)^{2} \int_{0}^{1} \rho^{1-k}(1-\rho)^{k} d \rho=t^{1-k}(1-t)^{k-2} B(2-k, 1+k) f_{-}(x)^{2}= \\
& \frac{B(2-k, 1+k)}{(k-1)^{k-1}(2-k)^{2-k}} f_{-}(x)^{2},
\end{aligned}
$$

for the optimal $t=k-1 \in(0,1)$. If $f(x)>0$, then $(f(x)+(1-t) r)_{-}=0, f_{-}(x)=0$ and the above equality holds formally.

Hence, we obtain

$$
\sum_{\eta_{j} \leq 0}\left|\eta_{j}\right| \leq \frac{1}{\pi^{2}} \frac{B(1, k-1) B(2-k, 1+k)}{(k-1)^{k-1}(2-k)^{2-k}} \int_{T^{2}} f(x)_{-}^{2} d x, \quad k \in(1,2),
$$

which proves (A.9) with

$$
\mathrm{L}_{1}\left(T^{2}\right) \leq \frac{1}{\pi^{2}} \min _{1<k<2} \frac{B(1, k-1) B(2-k, 1+k)}{(k-1)^{k-1}(2-k)^{2-k}} \leq \frac{1}{\pi^{2}} \frac{B(1,1 / 2) B(1 / 2,5 / 2)}{1 / 2}=\frac{3}{2 \pi} .
$$

The minimum is actually attained at $k=1.38 \ldots$, which gives $\mathrm{L}_{1}\left(T^{2}\right) \leq 0.459 \ldots$.

The inequality for the negative trace (A.9) is equivalent to the following inequalities for families of orthonormal functions and vector fields.

TheOREM A.3. Let a family of functions $\varphi_{1}, \ldots, \varphi_{m} \in H^{1}\left(T^{2}\right)$ be orthonormal in $L_{2}\left(T^{2}\right): \int_{T^{2}} \varphi_{i}(x) \varphi_{j}(x) d x=\delta_{i j}$, and let $\int_{T^{2}} \varphi_{j}(x) d x=0$. Then the following inequality holds:

$$
\int_{T^{2}} \rho(x)^{2} d x \leq \mathrm{k}_{2} \sum_{j=1}^{m}\left\|\nabla \varphi_{j}\right\|^{2}, \quad \mathrm{k}_{2} \leq \frac{6}{\pi}
$$

where $\rho(x)=\sum_{j=1}^{m} \varphi_{j}(x)^{2}$.

If a family of vector fields $u_{1}(x), \ldots, u_{m}(x) \in\left(H^{1}\left(T^{2}\right)\right)^{2}$ forms an orthonormal family in $\left(L^{2}\left(T^{2}\right)\right)^{2}, \int_{T^{2}} u_{i}(x) \cdot u_{j}(x) d x=\delta_{i j}$, and $\int_{T^{2}} u_{j}(x) d x=0$, then

$$
\begin{array}{r}
\int_{T^{2}} \rho(x)^{2} d x \leq \mathrm{k}_{2}^{\mathrm{vec}} \sum_{j=1}^{m}\left(\left\|\nabla u_{j}^{1}\right\|^{2}+\left\|\nabla u_{j}^{2}\right\|^{2}\right)=\mathrm{k}_{2}^{\mathrm{vec}} \sum_{j=1}^{m}\left(\left\|\operatorname{rot} u_{j}\right\|^{2}+\left\|\operatorname{div} u_{j}\right\|^{2}\right), \\
\mathrm{k}_{2}^{\mathrm{vec}} \leq \frac{12}{\pi},
\end{array}
$$


where $\rho(x)=\sum_{j=1}^{m}\left|u_{j}(x)\right|^{2}$. If, in addition, $\operatorname{div} u_{j}=0\left(\right.$ or $\left.\operatorname{rot} u_{j}=0\right)$, then

$$
\int_{T^{2}} \rho(x)^{2} d x \leq \begin{cases}\mathrm{k}_{2}^{\mathrm{sol}} \sum_{j=1}^{m}\left\|\operatorname{rot} u_{j}\right\|^{2}=\mathrm{k}_{2}^{\mathrm{sol}} \sum_{j=1}^{m}\left\|\nabla u_{j}\right\|^{2}, & \operatorname{div} u_{j}=0, \\ \mathrm{k}_{2}^{\mathrm{pot}} \sum_{j=1}^{m}\left\|\operatorname{div} u_{j}\right\|^{2}=\mathrm{k}_{2}^{\mathrm{pot}} \sum_{j=1}^{m}\left\|\nabla u_{j}\right\|^{2}, & \operatorname{rot} u_{j}=0,\end{cases}
$$

where

$$
\mathrm{k}_{2}^{\mathrm{sol}}=\mathrm{k}_{2}^{\mathrm{pot}} \leq \frac{\mathrm{k}_{2}^{\mathrm{vec}}}{2} \leq \frac{6}{\pi}
$$

Proof. We consider the Schrödinger operator acting on vector functions $u=\left(u^{1}, u^{2}\right)^{T}$ :

$$
-\Delta u+\Pi f \Pi u=-\left(\begin{array}{c}
\Delta u^{1} \\
\Delta u^{2}
\end{array}\right)+\left(\begin{array}{l}
\Pi\left(f \Pi u^{1}\right) \\
\Pi\left(f \Pi u^{2}\right)
\end{array}\right)
$$

with spectrum $-\infty<r_{1}(f) \leq r_{2}(f) \leq \ldots$. Since, for an eigenvalue $\eta$ of the operator (A.8) with eigenfunction $\varphi$, there corresponds the eigenvalue $r=\eta$ of multiplicity two with linearly independent eigenfunctions $(\varphi, 0)^{T}$ and $(0, \varphi)^{T}$, we obtain the following estimate for the negative trace of the operator (A.16):

$$
\sum_{r_{j}<0}\left|r_{j}\right| \leq \mathrm{L}_{1}^{\mathrm{vec}}\left(T^{2}\right) \int_{T^{2}} f^{2}(x) d x, \quad \text { where } \quad \mathrm{L}_{1}^{\mathrm{vec}}\left(T^{2}\right)=2 \mathrm{~L}_{1}\left(T^{2}\right) .
$$

We can now use the general result in [27] (see also [8], [35]) stating that the estimates for the negative trace (A.9), (A.17) of the operators (A.8), (A.16) are equivalent to the inequalities for orthonormal families (A.13), (A.14), respectively, and, in addition, in the two-dimensional case, the best constants are related by the equality

$$
\mathrm{k}_{2}=4 \mathrm{~L}_{1}\left(T^{2}\right) \quad \text { and } \quad \mathrm{k}_{2}^{\mathrm{vec}}=4 \mathrm{~L}_{1}^{\mathrm{vec}}\left(T^{2}\right),
$$

which proves (A.13) and (A.14).

There remains to prove (A.15). For $u(x)=\left(u^{1}(x), u^{2}(x)\right)^{T}$, we set

$$
\hat{u}(x)=\left(-u^{2}(x), u^{1}(x)\right)^{T} \text {. }
$$

It is easy to see that

$$
|u(x)|=|\hat{u}(x)|, \quad \operatorname{div} u(x)=\operatorname{rot} \hat{u}(x), \quad \operatorname{rot} u(x)=-\operatorname{div} \hat{u}(x) .
$$

Furthermore, if $u_{1}, \ldots, u_{m}$ are orthonormal in $\left(L_{2}(\Omega)\right)^{2}$, then $\hat{u}_{1}, \ldots, \hat{u}_{m}$ are orthonormal and vice versa. This shows that $\mathrm{k}_{2}^{\text {sol }}=\mathrm{k}_{2}^{\text {pot }}$. Let us prove the inequality $\mathrm{k}_{2}^{\mathrm{sol}} \leq \mathrm{k}_{2}^{\mathrm{vec}} / 2$. Suppose that the family $u_{1}, \ldots, u_{m}$ is orthonormal in $\left(L_{2}\left(T^{2}\right)\right)^{2}$ and let $\operatorname{div} u_{j}=0$ for $j=1, \ldots, m$. We set $\rho(x)=\sum_{j=1}^{m}\left|u_{j}(x)\right|^{2}$ and consider the family of $2 m$ vector functions $u_{1}, \ldots, u_{m}, \hat{u}_{1}, \ldots, \hat{u}_{m}$. Since $\operatorname{rot} \hat{u}_{j}=0, j=1, \ldots m$, it follows that $\left(u_{i}, \hat{u}_{j}\right)=0$ for $1 \leq i, j \leq m$ and this family is orthonormal. Applying (A.14) to this family of $2 m$ functions, we obtain

$$
\begin{aligned}
& 4 \int_{T^{2}} \rho(x)^{2} d x=\int_{T^{2}}\left(\sum_{j=1}^{m}\left(\left|u_{j}(x)\right|^{2}+\left|\hat{u}_{j}(x)\right|^{2}\right)\right)^{2} d x \leq \\
& \mathrm{k}_{2}^{\operatorname{vec}} \sum_{j=1}^{m}\left(\left\|\operatorname{rot} u_{j}\right\|^{2}+\left\|\operatorname{div} \hat{u}_{j}\right\|^{2}\right)=2 \mathrm{k}_{2}^{\operatorname{vec}} \sum_{j=1}^{m}\left\|\operatorname{rot} u_{j}\right\|^{2} .
\end{aligned}
$$


Thus, $\mathrm{k}_{2}^{\mathrm{sol}} \leq \mathrm{k}_{2}^{\mathrm{vec}} / 2$ and the proof is complete.

Acknowledgments. This work was initiated while A.A.I. was visiting A.M. at the University of Poitiers, France.

This work was supported in part by the US Civilian Research and Development Foundation, grant no. RM1-2343-MO-02 ( A.A.I. and E.S.T.), by INTAS, grant no. 00899 (A.A.I and A.M.) and by the Russian Foundation for Fundamental Research, grants no. 03-01-00189 and no. 02-01-00801 (A.A.I.). The work of E.S.T. was supported in part by the National Science Foundation, grant no. DMS-0204794, the MAOF Fellowship of the Israeli Council of Higher Education, and by the Department of Energy under contract W-7405-ENG-36 and the ASCR Program in Applied Mathematical Sciences.

\section{REFERENCES}

[1] Araki H., On an inequality of Lieb and Thirring, Lett. Math. Phys., 19, 167-170, 1990.

[2] Babin A.V. and Vishik M.I., Attractors of Evolution Equations, Nauka, Moscow, English transl, 1988, North-Holland, Amsterdam, 1992.

[3] Barcilon V., Constantin P., and Titi E.S., Existence of solutions to the Charney model of the gulf stream, SIAM J. Math. Anal., 19, 1355-1364, 1988.

[4] Cao C., Rammaha M. A., and Titi E.S. The Navier-Stokes equations on the rotating 2-D sphere: Gevrey regularity and asymptotic degrees of freedom, Z. angew. Math. Phys., 50, 341-360, 1999.

[5] Bessiah H. and Flandoli F., Weak attractor for a dissipative Euler equation, J. Dynam. Differential Equations, 12, 713-732, 2000.

[6] Chen Z. M., Ghil M., Simonnet E., and Wang S., Hopf bifurcation in quasi-geostrophic channel flow, SIAM J. Appl. Math., 64(1), 343-368, 2004.

[7] Chepyzhov V.V. and Ilyin A.A., A note on the fractal dimension of attractors of dissipative dynamical systems, Nonlinear Anal., 44, 811-819, 2001.

[8] Chepyzhov V.V. and Ilyin A.A., On the fractal dimension of invariant sets; applications to Navier-Stokes equations, Discrete and Continuous Dynamical Systems, 10, 117-135, 2004.

[9] Cockburn B., Jones D. and Titi E.S., Estimating the number of asymptotic degrees of freedom for nonlinear dissipative systems, Mathematics of Computation, 66, 1073-1087, 1997.

[10] Constantin P. and Foias C., Global Lyapunov exponents, Kaplan-Yorke formulas and the dimension of the attractors for the 2D Navier-Stokes equations, Comm. Pure Appl. Math., $38,1-27,1985$.

[11] Constantin P. and Foias C., Navier-Stokes Equations, Univ. of Chicago Press, Chicago, 1988.

[12] Constantin P., Foias C., and Temam R., On the dimension of the attractors in two-dimensional turbulence, Physica D, 30, 284-296, 1988.

[13] Dymnikov V.P. and Filatov A.N., Mathematics of Climate Modelling, Boston, Birkhäuser, 1997.

[14] Foias C., Manely O., Rosa R. and Temam R., Navier-Stokes Equations and Turbulence, Cambridge, UK, Cambridge Univ. Press, 2001

[15] Foias C. and Prodi G., Sur le comportement global des solutions non stationnaires des équations de Navier-Stokes en dimension two, Rend. Sem. Mat. Univ. Padova, 39, 1-34, 1967.

[16] Foias C. and Temam R., Determination of the solutions of the Navier-Stokes equations by a set of nodal values, Mathematics of Computation, 43, 117-133, 1984.

[17] Hale J., Asymptotic Behavior of Dissipative Systems, Providence, RI, Amer. Math. Soc., 1988.

[18] Hauk S., The Long-term Behavior of the Stommel-Charney Model of the Gulf Stream, an Analytical and Computational Study, Ph.D. Thesis, Department of Mathematics, University of California, Irvine, 1997.

[19] Hauk S. and Titi E.S., Long-time behavior of the Stommel-Charney model of the gulf stream, Preprint.

[20] Ilyin A.A., Lieb-Thirring inequalities on the $N$-sphere and in the plane, and some applications, Proc. London Math. Soc., 67, 159-182, 1993.

[21] Ilyin A.A., The Euler equations with dissipation, Mat. Sbornik, 182, no. 12, 1729-1739, 1991 English transl. in Math. USSR, Sbornik, 74, no. 2, 475-486, 1993.

[22] Ilyin A.A., Partly dissipative semigroups generated by the Navier-Stokes system on two- 
dimensional manifolds and their attractors, Mat. Sbornik, 184, no. 1, 55-88, 1993. English transl. in Russ. Acad. Sci. Sb. Math., 78, no. 1, 47-76, 1993.

[23] Ilyin A.A. and Titi E.S., Attractors to the two-dimensional Navier-Stokes- $\alpha$ models: an $\alpha-$ dependence study, J. Dynam. Differential Equations, 15, 751-778, 2003.

[24] Jones D. and Titi E.S., On the number of determining nodes for the 2-D Navier-Stokes equations, Journal of Mathematical Analysis \& Applications, 168, 72-88, 1992.

[25] Jones D. and Titi E.S., Upper bounds on the number of determining modes, nodes, and volume elements for the Navier-Stokes equations, Indiana University Mathematics Journal, 42, 875-887, 1993.

[26] Ladyzhenskaya O.A., Attractors for Semigroups and Evolution Equations, Leizioni Lincei, Cambridge Univ. Press, Cambridge, 1991.

[27] Lieb E. and Thirring W., Inequalities for the moments of the eigenvalues of the Schrödinger Hamiltonian and their relation to Sobolev inequalities, Studies in Mathematical Physics. Essays in honor of Valentine Bargmann, Princeton University Press, 269-303, 1976.

[28] Liu V.X., A sharp lower bound for the Hausdorff dimension of the global attractors of the $2 D$ Navier-Stokes equations, Comm. Math. Phys., 158, 327-339, 1993.

[29] Meshalkin L.D. and Sinai Ya. G., Investigation of the stability of a stationary solution of a system of equations for the plane movement of an incompressible viscous liquid, Prikl. Mat. Mekh., 25, 1140-1143, 1961. English transl. in J. Appl. Math. Mech., 25, 1961.

[30] Pedlosky J., Geophysical Fluid Dynamics, New York, Springer, 1979.

[31] Robinson J.C., Infinite Dimensional Dynamical Systems, Cambridge, Cambridge University Press, 2001.

[32] Saut J.-C., Remarks on the damped stationary Euler equations, Diff. Int. Equations, 3, 801-812, 1990.

[33] Sell G.R. and You Y., Dynamics of Evolutionary Equations, New York, Springer-Verlag, 2002.

[34] Temam R., Navier-Stokes Equations. Theory and Numerical Analysis, Amsterdam, NorthHolland, 1984.

[35] Temam R., Infinite Dimensional Dynamical Systems in Mechanics and Physics, 2nd ed., New York, Springer-Verlag, 1997.

[36] Wolansky G., Existence, uniqueness, and stability of stationary barotropic flow with forcing and dissipation, Comm. Pure Appl. Math., 41, 19-46, 1988.

[37] Yudovich V.I., Example of the generation of a secondary stationary or periodic flow when there is loss of stability of the laminar flow of a viscous incompressible fluid, Prikl. Mat. Mekh., 29, 453-467, 1965. English transl. in J. Appl. Math. Mech., 29, 1965.

[38] Ziane, M., Optimal bounds on the dimension of attractors for the Navier-Stokes equations, Physica D, 105, 1-19, 1997. 